\title{
Rendimiento estructural de bloques de hormigón con agregados de madera para la construcción de edificios de mediana y gran altura
}

\author{
Structural performance of concrete blocks with wood aggregates for the \\ construction of medium and high-rise buildings
}

\author{
David Dominguez-Santos ${ }^{(*)}$
}

\section{RESUMEN}

Los bloques de hormigón son elementos muy utilizados en edificación, debido a su bajo coste, su buen desempeño y la facilidad de montaje que tiene en los muros. Sin embargo, la baja ductilidad y resistencia a la flexión, podría perjudicar el comportamiento sismorresistente de las edificaciones, sobre todo en las construcciones de mediana y gran altura. Sin embargo, el añadido de aditivos reciclados y abundantes en la Naturaleza como la madera podrían mejorar las características estructurales desde el punto de vista sísmico y estructural. Este trabajo analiza y compara el rendimiento y el desempeño estructural que adquieren los bloques de hormigón ordinario incorporando en su elaboración diferentes tipos de aditivos de madera (desechos de aserrín y virutas de madera) en pórticos de mediana y gran altura (4, 8, 12 y 16 plantas). La modelación de los pórticos, utilizarán resultados experimentales realizados en un laboratorio de la Universidad de Talca (Chile).

Palabras clave: Comportamiento sismorresistente; Materiales; Estructuras; Hormigón; Aserrín; Virutas de madera; Bloques.

\section{ABSTRACT}

Concrete blocks are widely used elements in buildings, due to their low cost, good performance and ease of mounting on the walls. However, the low ductility and resistance to flexion, could harm the seismic-resistant behavior of buildings, especially in medium and high-rise buildings. However, the addition of recycled and abundant additives in Nature such as wood could improve the structural and seismic characteristics. This work analyzes and compares the performance and structural performance of ordinary concrete blocks, incorporating different types of wood additives (sawdust and wood shaving) in medium and high-height frames (4, 8, 12 and 16 stories). The modeling of the frames will use experimental results carried out in a laboratory at the University of Talca (Chile).

Keywords: Seismic-resistant behavior; Materials; Structures; Concrete; Sawdust; Wood shaving; Blocks.

(*) Department of Engineering and Building Management, University of Talca, Curicó (Chile).

Persona de contacto/Corresponding author: ddominguez@utalca.cl (David Dominguez Santos).

ORCID: https://orcid.org/oooo-ooo1-6664-9011 (D. Dominguez).

Cómo citar este artículo/Citation: David Dominguez-Santos (2021). Rendimiento estructural de bloques de hormigón con agregados de made-
ra para la construcción de edificios de mediana y gran altura. Informes de la Construcción, 73(564): e414. https://doi.org/10.3989/ic.81319

Copyright: (c) 2021 CSIC. Este es un artículo de acceso abierto distribuido bajo los términos de la licencia de uso y distribución Creative Commons Reconocimiento 4.0 Internacional (CC BY 4.0). 


\section{INTRODUCCIÓN}

La construcción es el causante del 20\% de las emisiones de efecto invernadero (1). Es por ello, que durante los últimos años se haya tratado de generar materiales sostenibles en el medio ambiente, reduciendo los efectos contaminantes existentes en el mundo y a su vez que reduzcan los costes en la construcción, favoreciendo a la sociedad.

Por otra parte, otro de los aspectos importantes es la mejora del rendimiento estructural de las edificaciones, derivado de las exigencias de la sociedad provocadas por los avances tecnológicos de los sistemas en construcción (disipadores, aisladores, muros, riostras) y los nuevos y modificados materiales utilizados en construcción (2), necesarias debido a las catástrofes producidos durante los últimos 50 años referentes a los terremotos y los agentes climatológicos, derivados del cambio climático.

La versatilidad del hormigón en las estructuras, debido a las propiedades mecánicas, la rapidez y la facilidad de montaje que tiene este material, haya hecho que sea uno de los materiales más utilizados en la construcción. Es por ello, que en muchos países ubicados en zonas sísmicas altamente exigentes como Turquía (3), Nepal (4), Perú (5) y Chile $(6,7)$, se haya utilizado mayoritariamente como solución estructural para reducir la vulnerabilidad sísmica de las edificaciones. Sin embargo, las características dúctiles y térmicas que tiene este material, disminuye las condiciones energéticas de las edificaciones, sobre todo cuando es usado en los muros.

Las características que tiene este material en los muros de relleno, ha hecho que durante la última década, se hayan realizado numerosos estudios con la finalidad de mejorar sus propiedades. Entre todos los estudios existentes, se ha demostrado la eficiencia que tiene la introducción de elementos compuestos de hormigón en los muros de relleno $(8,9)$ en edificaciones porticadas de baja altura, sin embargo, existe desconocimiento de su comportamiento en edificaciones de mayor altura.

El objetivo de este trabajo es contribuir al uso de materiales ligeros en edificaciones de mediana y gran altura, que puedan impactar positivamente en las políticas de ahorro de energía, la sostenibilidad en la fabricación, el reciclaje de residuos (10) y la mejora de algunas de las propiedades estructurales sismorresistente (flexión, ductilidad y disminución de pesos) de las edificaciones, utilizando materias primas obtenidas de residuos agrícolas y forestales (aserrín o viruta) que se producen en grandes cantidades y que son eliminados en vertederos sin ninguna revalorización, sin alterar las propiedades de los materiales y elementos tradicionales.

\section{ANTECEDENTES}

La utilización de muros en las edificaciones porticadas ha sido una de las soluciones más empleadas para mejorar las condiciones estructurales en el mundo de la construcción.

Los materiales mayormente utilizados en los muros y en la construcción son los hormigones, el cemento y la arcilla, elementos que han demostrado su eficacia y su eficiencia en las edificaciones.
Durante los últimos años, estos materiales han sido objeto de numerosos estudios e investigaciones, con la finalidad de mejorar algunas de sus propiedades y características. Para ello, la introducción de diferentes aditivos en su fabricación, ha sido el detonante para mejorar sus propiedades más deficitarias.

En la literatura científica, hay abundantes estudios sobre la introducción de aditivos incorporados al hormigón y al cemento tradicional. Son ejemplos, la adición de fibras metálicas o de polipropileno a la mezcla que aumentan las propiedades de capacidad de carga (11-13), de resistencia al corte o la ductilidad (14). Asimismo, pero desde un punto de vista ambiental (daños causados por la extracción de materias primas y/o las emisiones de $\mathrm{CO} 2$ ), algunas mezclas han considerado la adición de cenizas volcánicas (15), madera (16), hormigón reciclado (17-19), entre otros.

Estos materiales, aparecen con mayor o menor relevancia en diferentes elementos constructivos en los edificios. Entre ellas, los bloques de hormigón y los ladrillos de arcilla, que son utilizados mayoritariamente en los muros de cerramiento (estructurales y no estructurales) y en particiones interiores debido a su coste y a sus buenas características constructivas.

Debido a ello, varios investigadores han estudiado la influencia de aditivos en estos elementos, con la finalidad de mejorar las propiedades térmicas $(20,21)$, acústicas (22), mecánicas (23), entre otros.

Se ha demostrado que la utilización de muros en las edificaciones porticadas de baja altura mejora el comportamiento sísmico de las edificaciones, debido a la mayor rigidez y resistencia elástica inicial que infringen estos elementos en las estructuras $(24,25,26)$. Sin embargo, esta afirmación no es tan relevante en las edificaciones de mediana y gran altura, debido a la gran rigidez y al peso que proporcionan estos elementos a las estructuras. Para solucionar estos problemas, estos elementos han sido sustituidos por otros más complejos que no dependan de estas características; disipadores, riostras, aisladores de base, entre otros. Estos elementos, han demostrado un buen rendimiento en las edificaciones, pero la complejidad, los costos y la preparación que requiere su colocación en las construcciones por parte de los trabajadores, se ha visto afectado en su utilización en las estructuras.

Debido a ello, los muros tradicionales siguen siendo la solución más utilizada en la construcción. Es por ello, que diferentes materiales se hayan introducido como aditivos a estos elementos (bloques) que componen los muros, con la finalidad de mejorar alguna de sus propiedades.

Entre los diferentes materiales que se pueden usar como aditivos en el cemento está la madera, que tiene propiedades interesantes desde el punto de vista sísmico y mecánico (27-29, 24), funciona bien cuando está flexionado y comprimido (30), y tiene una baja conductividad térmica (31), acústica y eléctrica (Norma ASTM D143-09 1927 (31); Wangaard 1966 (32); Hearmon 1948 (33)).

Destacar que países sísmicos como Chile, Colombia y otros países del sudeste asiático, tienen grandes reservas de ma- 
dera $(34,35)$. Estos países procesan este material para crear múltiples productos derivados, lo que significa que tienen grandes volúmenes de desechos de madera en fábricas y aserraderos que podrían ser potencialmente utilizados para otros fines (36), como en la construcción.

\section{MATERIALES Y MÉTODOS UTILIZADOS}

\subsection{Consideraciones previas}

Es evidente que la dosificación de aditivos en las mezclas de hormigón determina las propiedades finales que adquiere (por ejemplo, la resistencia, la ductilidad y la trabajabilidad).

Este estudio, continua la investigación empezada por D. Dominguez et al. (24) para edificaciones de altura. Para ello, se utiliza los resultados mecánicos de compresión y flexo-tracción realizados a los bloques de hormigón con aserrín y viruta obtenidos con las máquinas hidráulicas del laboratorio de construcción de la Facultad de Ingeniería de la Universidad de Talca.

La fabricación del hormigón utilizado en los bloques, siguió las especificaciones de las normas chilenas (37-40) y españolas $(41,42)$.

Las mezclas utilizadas para la fabricación de los bloques de hormigón, reemplazaron parte de la grava por aditivos de madera (aserrín o virutas). Para este estudio, solo se consideró la utilización de bloques ordinarios y bloques con la incorporación de un $10 \%$ de aditivo de madera en la preparación de las mezclas, debido a su uso estructural y a sus propiedades resistentes.

Los bloques utilizados fueron sometidos a ensayos de compresión y flexo-compresión según la Norma chilena NCh 181. Para cada muestra, se determinó su capacidad mecánica, mediante las curvas de compresión y flexo-compresión.

Tal y como se indica en (24), se ha buscado el respeto por el medio ambiente y la sostenibilidad, utilizando productos abundantes existentes en lugares altamente sísmicos como Chile y Japón. Es por ello, que en esta investigación y en (24), se utilizarán los desechos de industrias y fábricas madereras (en forma de aserrín y viruta), procedentes de diferentes especies de árboles, para incluir en las mezclas que componen los bloques. Los detalles de las mezclas y de las granulometrías, se especificaron en (24). Para la modelización de los muros de mampostería teniendo en cuenta las juntas de unión entre mortero y bloque, se ha utilizado $(24,25,43,44-47)$.

\subsection{Resultados mecánicos utilizados}

La resistencia a la compresión promedio de todos los bloques de hormigón realizado en (24), se midió a los 7, 14 y 28 días después de su elaboración. Como se dijo previamente, este estudio solo contempla los resultados aptos para su uso estructural según la Norma Chilena NCh 181 (2006), es decir, los bloques de hormigón tradicionales y los bloques de hormigón con el 10\% de agregados de aserrín y viruta. Los valores medios de los ensayos de laboratorio, utilizados para esta investigación aparecen en la tabla 1.
Tabla 1. Resultados mecánicos de los bloques (24).

\begin{tabular}{|l|c|c|c|}
\hline Tipo de bloque & $\begin{array}{c}\text { Compresión } \\
\left(\mathbf{N} / \mathbf{m m}^{2}\right)\end{array}$ & $\begin{array}{c}\text { Flexo-tracción } \\
\left(\mathbf{N} / \mathbf{m m}^{2}\right)\end{array}$ & $\begin{array}{c}\text { Peso } \\
\text { bloque } \\
(\mathbf{N})\end{array}$ \\
\hline o\% (Ordinario) & 1.47 & 0.31 & 160.02 \\
\hline Con el 10\% Aserrín & 1.28 & 0.34 & 136.12 \\
\hline Con el 10\% Viruta & 1.20 & 0.33 & 135.55 \\
\hline
\end{tabular}

Los valores obtenidos en la tabla 1 provienen de las curvas de capacidad de compresión y flexo-tracción de los bloques de hormigón realizados a los 28 días después de su fabricación. Estas curvas fueron obtenidas de acuerdo con la Norma chilena NCh 1037.

\section{ANÁLISIS ESTRUCTURAL}

Esta sección describe el comportamiento sísmico de modelos estructurales representativos de zonas medianamente sísmicas utilizando análisis estáticos no lineales (Push-over). Los cálculos se implementaron con el software de análisis estructural Seismostruct v.7.0.2. Este software, se basa en un análisis de elementos finitos, producto de la empresa Seismosoft $®$ (48). Este software, permite estimar la relación entre el desplazamiento en la última planta y el cortante máximo en la base de los edificios bajo cargas estáticas y dinámicas, considerando el comportamiento de los materiales no lineales en todas sus geometrías. Los resultados de estos análisis estático no lineales (Push-over), se muestran en las respectivas curvas de capacidad.

\subsection{Descripción de los pórticos analizados}

Los modelos analizados en este trabajo se basan en estructuras porticadas, debido a la abundancia de este sistema de construcción en muchos países latinoamericanos y europeos $(25,26)$. Estas estructuras, se caracterizan por tiempos de ejecución rápidos y pocos recursos materiales.

Tradicionalmente, el comportamiento estructural de los edificios hechos de vigas y columnas sin ningún tipo de dispositivo antisísmico durante los terremotos no ha sido satisfactorio. En consecuencia, es necesario incorporar muros $\mathrm{u}$ otros elementos resistentes con la intención de disminuir los posibles daños ocasionados $(25,26)$, cumpliendo con las exigencias Normativas.

En los últimos años, la mayoría de los países europeos han estado adaptando sus códigos nacionales (estructurales) para parecerse a los códigos europeos (Eurocódigos) y americanos. En consecuencia, los resultados de este trabajo pueden considerarse representativos para un porcentaje significativo de edificios existentes en Europa y parte de América Latina.

Como se muestra en la figura 1, cada modelo de pórtico está formado por 4 vanos de 5 metros de longitud (esta medida es a ejes de pilares). De los 4 vanos que conforma cada pórtico, 2 de ellos está relleno de muros de bloques, mientras que los otros 2 se dejan vacíos. Los vanos vacíos, simularían a las ventanas y puertas que tendría la composición arquitectónica de los edificios.

El diseño de los elementos de hormigón armado de estos pórticos (vigas y pilares), han sido diseñados considerando 
la Normativa americana ACI-318 y la Normativa española EHE-o8, sin considerar las fuerzas sísmicas y sin considerar los muros de relleno.

Los bloques que conforman los muros de relleno de los pórticos se ubican por debajo de la viga superior de cada planta sin ningún anclaje a los elementos estructurales.

La altura entre pisos es de tres metros, con a una altura libre por planta de 2.60 metros. Esto significa, que los modelos utilizados tienen una altura de 12, 24, 36 y 48 metros para los pórticos de 4, 8, 12 y 16 alturas, respectivamente. La configuración de los pórticos es regular y simétrica en elevación (Figura 1). Estos pórticos, cuyos muros de relleno se ubican en los vanos externos, corresponderían a las fachadas externas de los edificios.

Los elementos estructurales horizontales de los pórticos corresponden a vigas de $30 \mathrm{~cm}$ de ancho y $40 \mathrm{~cm}$ de alto en todas las alturas. Los elementos verticales de cada pórtico están formados por cinco columnas cuadradas que varían en $10 \mathrm{~cm}$ de lado (X, Y) cada 4 plantas. Las cuatro plantas superiores de los modelos corresponden a columnas de $30 \times 30 \mathrm{~cm}$. La composición de las medidas de las columnas aparece detallada en la Figura 1. La continuidad estructural de los elementos

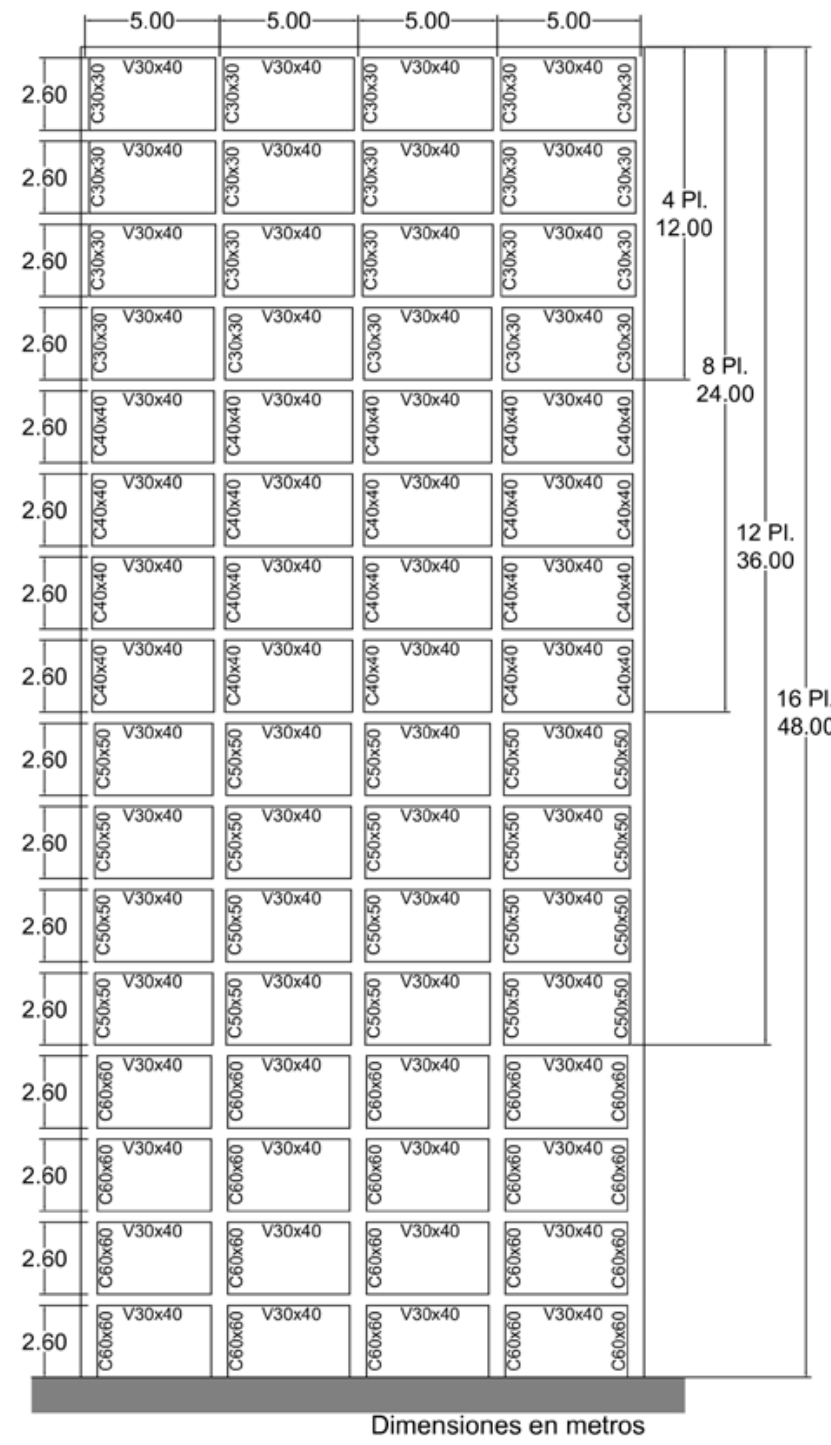

Figura 1. Dimensiones y geometría de los pórticos. que conforman los pórticos se logra a través de las armaduras longitudinales, correspondientes a varillas de acero que conectan las vigas y columnas, cumpliendo con todos los requerimientos de los códigos europeos y americanos de diseño estructural.

Los muros de relleno no estructurales están hechos de bloques de hormigón de $19.1 \mathrm{~cm}$ espesor, separados por mortero de $1 \mathrm{~cm}$ de espesor y con refuerzos transversales de acero tipo escalera cada cuatro líneas de bloques (Figura 2).

Las características y propiedades más importantes de los modelos se resumen en la Tabla 2. Los materiales utilizados para los elementos estructurales (vigas y columnas), fueron: hormigón HA-30 correspondiente con una resistencia característica de $f_{c k}=29.42 \mathrm{~N} / \mathrm{mm}^{2}$ y acero B-50o-S, con un límite elástico del acero $f_{y k}=490.33 \mathrm{~N} / \mathrm{mm}^{2}$. Ambos materiales están definidos en la Norma CTE DB SE AE.
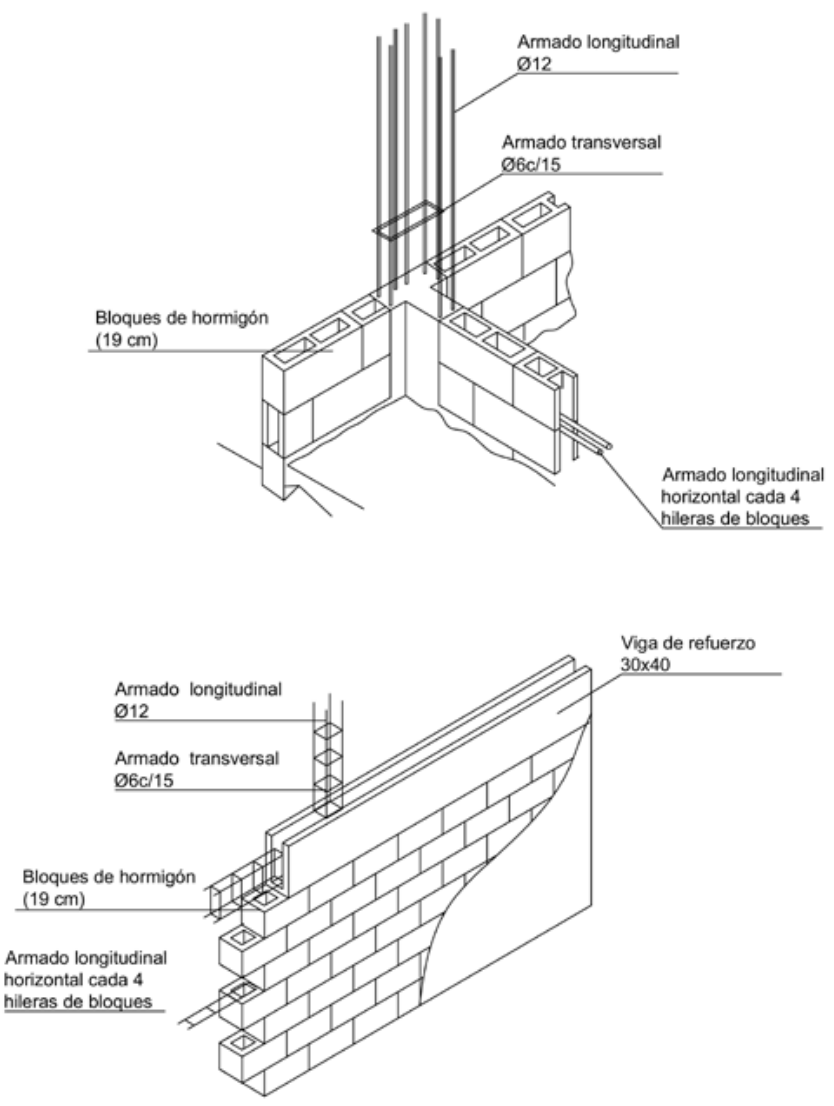

Figura 2. Colocación de bloques.

En la Tabla 2, cada pórtico se denota con un número y letras. El primer número indica la cantidad de alturas que tiene el pórtico y las letras, el tipo de elemento utilizado en los muros de relleno: "NW" significa pórtico sin muros, "WTB" significa pórtico con bloques tradicionales, "WAB" significa pórtico con bloques con agregado de aserrín y "WVB" significa pórtico con agregados de viruta de madera. Esta tabla muestra la altura y la longitud total del pórtico, las dimensiones de las vigas y pilares, el peso del pórtico siguiendo la combinación de acciones $\mathrm{G}+$ o.3Q según el Eurocódigo 8, y los períodos fundamentales de los diferentes modelos analizados.

En la combinación de acciones que determina el peso del pórtico, G determina el peso propio de la estructura y $\mathrm{Q}$ 
Tabla 2. Configuración de los pórticos.

\begin{tabular}{|c|c|c|c|c|c|c|}
\hline $\begin{array}{l}\text { Tipo de } \\
\text { Pórtico } \\
\end{array}$ & $\begin{array}{l}\text { Altura total } \\
(\mathbf{m})\end{array}$ & $\begin{array}{l}\text { Longitud } \\
\text { total (m) }\end{array}$ & $\begin{array}{l}\text { Vigas } \\
\left(\mathrm{cm}^{2}\right)\end{array}$ & $\begin{array}{c}\text { Pilares } \\
\left(\mathrm{cm}^{2}\right)\end{array}$ & $\begin{array}{c}\text { Peso total } \\
(\mathbf{k N})\end{array}$ & $\begin{array}{c}\text { Periodo } \\
\text { fundamental (s) }\end{array}$ \\
\hline $4-\mathrm{NW}$ & 12 & 20.5 & $30 \times 40$ & $30 \times 30$ & 2691 & 0.56 \\
\hline $4-$ WTB & 12 & 20.5 & $30 \times 40$ & $30 \times 30$ & 3273 & 0.32 \\
\hline $4-W A B$ & 12 & 20.5 & $30 \times 40$ & $30 \times 30$ & 3239 & 0.35 \\
\hline $4-W V B$ & 12 & 20.5 & $30 \times 40$ & $30 \times 30$ & 3228 & 0.36 \\
\hline $8-\mathrm{NW}$ & 24 & 20.5 & $30 \times 40$ & $30 \times 30 / 40 \times 40$ & 5485 & 0.88 \\
\hline $8-$ WTB & 24 & 20.5 & $30 \times 40$ & $30 \times 30 / 40 \times 40$ & 6650 & 0.62 \\
\hline $8-W A B$ & 24 & 20.5 & $30 \times 40$ & $30 \times 30 / 40 \times 40$ & 6581 & 0.68 \\
\hline $8-$ WVB & 24 & 20.5 & $30 \times 40$ & $30 \times 30 / 40 \times 40$ & 6559 & 0.69 \\
\hline $12-\mathrm{NW}$ & 36 & 20.5 & $30 \times 40$ & $30 \times 30 / 40 \times 40 / 50 \times 50$ & 8410 & 1.24 \\
\hline $12-$ WTB & 36 & 20.5 & $30 \times 40$ & $30 \times 30 / 40 \times 40 / 50 \times 50$ & 10156 & 0.91 \\
\hline $12-\mathrm{WAB}$ & 36 & 20.5 & $30 \times 40$ & $30 \times 30 / 40 \times 40 / 50 \times 50$ & 10054 & 0.98 \\
\hline $12-\mathrm{WVB}$ & 36 & 20.5 & $30 \times 40$ & $30 \times 30 / 40 \times 40 / 50 \times 50$ & 10020 & 1.00 \\
\hline $16-\mathrm{NW}$ & 48 & 20.5 & $30 \times 40$ & $30 \times 30 / 40 \times 40 / 50 \times 50 / 60 \times 60$ & 11495 & 1.65 \\
\hline $16-$ WTB & 48 & 20.5 & $30 \times 40$ & $30 \times 30 / 40 \times 40 / 50 \times 50 / 60 \times 60$ & 13823 & 1.26 \\
\hline $16-\mathrm{WAB}$ & 48 & 20.5 & $30 \times 40$ & $30 \times 30 / 40 \times 40 / 50 \times 50 / 60 \times 60$ & 13686 & 1.31 \\
\hline $16-$ WVB & 48 & 20.5 & $30 \times 40$ & $30 \times 30 / 40 \times 40 / 50 \times 50 / 60 \times 60$ & 13641 & 1.32 \\
\hline
\end{tabular}

las cargas vivas, considerándose un uso residencial, administrativo o pequeño comercio, correspondiente a $2 \mathrm{kN} /$ $\mathrm{m}^{2}$ (49) en todas las plantas del pórtico a excepción del piso superior (techo), cuya carga fue de $1 \mathrm{kN} / \mathrm{m}^{2}$ (mantenimiento).

El presente trabajo, no tuvo en cuenta la colaboración de la carpintería de ventanas en las aberturas, debido a su gran fragilidad.

Por último, en cada una de las plantas que conforma el pórtico, se ha considerado un comportamiento rígido "diafragma", elemento que limita los posibles desplazamientos en el eje vertical.

\subsection{Modelo del pórtico}

Los elementos que componen cada uno de los pórticos fueron modelados utilizando elementos finitos de barra. Para cada elemento estructural (columnas y vigas), se siguieron las prescripciones propuestas por Mander (50) para el hormigón y el modelo bilineal de Ferrara (51) para barras de acero reforzado.

Además, las vigas y pilares existentes están representadas por elementos finitos de barra no lineales (52), donde las no linealidades se concentran en las rótulas plásticas ubicadas en los extremos de cada barra, correspondiente al 15\% de la longitud total del elemento (53). De acuerdo con Scott et al. (54), se consideró que las uniones/conexiones entre las columnas y vigas de hormigón eran rígidas, mientras que el comportamiento histerético que representa la distribución de tensiones, fueron calculadas con modelos de fibra basados en las propiedades del material y la geometría de los elementos estructurales (discretizado con 300 fibras). En el modelo, las cargas se han aplicado sobre las vigas.

Las tolerancias utilizadas para los desplazamientos y las rotaciones, fueron del orden de $10^{-5}$ en todos los casos con un número máximo de 300 iteraciones.
Para el análisis numérico, se utilizó el método Newmark- $\beta$, método de integración numérico fiable, ampliamente utilizado en dinámica de estructuras. En este método, los factores Beta $(\beta)$ y Gama $(\gamma)$, son coeficientes que dependen de la frecuencia natural (w) y la amortiguación (s) de la estructura. Para este trabajo, se utilizaron $\beta=0.25$ y $\gamma=0.5$, ya que con estos valores el método Newmark- $\beta$ es implícito y estable (55). Finalmente, se consideró el modelo de Rayleigh de $4 \%$ (modo 1) y 6\% (modo 2) (56). El método para la combinación modal utilizada en los análisis fue la combinación cuadrática completa (CQC) con una amortiguación correspondiente a $0.04(4 \%)$.

La simulación del comportamiento mecánico de cada material en los elementos del pórtico, requiere ingresar varios datos correspondientes a las propiedades del material. Es por ello, que los valores experimentales de plastificación y rotura obtenidos a partir de las curvas de capacidad de cada uno de los materiales se consideraron suficientes. Por otra parte, las deformaciones unitarias correspondientes a los procesos de falla del hormigón y acero usaron los valores estándar de Seismostruct (48): agrietamiento del hormigón (0.0001), desprendimiento del hormigón (-0.002), rotura del hormigón (-0,002), fluencia (0.0025) y fractura de acero (0.06). Además, los criterios referentes a la curvatura y las rotaciones, se verificaron a través de la capacidad de rotación de Mergos y Kappos (57) y a la capacidad de corte establecida en el Eurocódigo EC-8.

\subsection{Modelos de los muros de relleno}

La presencia de paneles de relleno modifica considerablemente el comportamiento estructural de las estructuras RC, haciéndolas más resistentes y rígidas. El modelado de la pared de relleno considera el comportamiento inelástico no lineal, la determinación de las propiedades mecánicas y la interacción con el pórtico. Sin embargo, hay muchas técnicas que permiten analizar los muros rellenos. En este sentido, Crisafulli et al. (43) realizó una revisión detallada con varias 
publicaciones sobre este tema. Después de realizar una revisión de la literatura, este trabajo adopta el enfoque de doble puntal propuesto por Crisafulli e implementado por Piestley et al. (58) utilizando el software Seismosoft®. El criterio principal considerado para la selección de este sistema de cálculo, fue el buen desempeño de los efectos de interacción panel-pórtico propuesto para estos modelos utilizados. Además, el enfoque de doble puntal se ha aplicado con éxito para la predicción de la respuesta sísmica de pórticos de hormigón armado de varias plantas, con resultados verificados (59).

El enfoque de Crisafulli, propone un macromodelo para la evaluación de la respuesta global de este sistema. El modelo se implementa como un elemento de panel de cuatro nodos, que está conectado al pórtico en las uniones de viga-columna (Figura 3). Internamente, el elemento del panel explica por separado el comportamiento de compresión y corte del muro de mampostería, utilizando dos puntales paralelos y un resorte de corte en cada dirección. Este modelo, permite considerar adecuadamente la rigidez lateral del panel y la resistencia del panel de mampostería, particularmente cuando se espera una falla de corte a lo largo de las juntas de mortero o una falla de tensión diagonal. Otros detalles numéricos sobre la transformación de las fuerzas en los nodos internos y ficticios a las fuerzas externas en los cuatro nodos se pueden seguir en (6o).
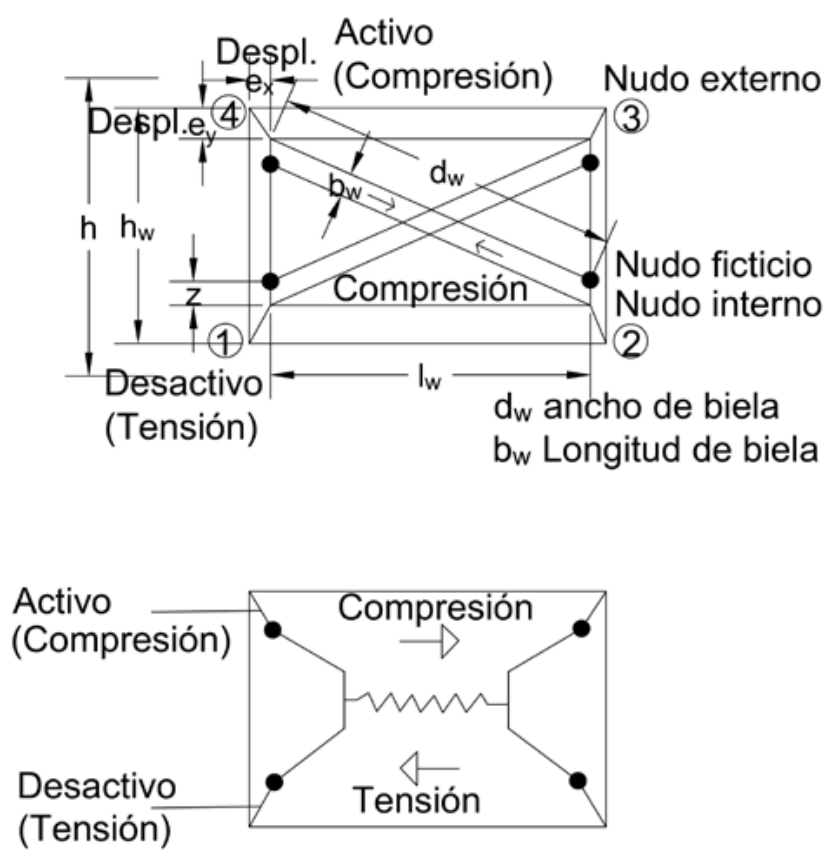

Figura 3. Modelos utilizados de muros de relleno.

\subsection{Caracterización de los muros de relleno}

Para la calibración del modelo utilizado en este trabajo para los muros de bloques, se han utilizado los parámetros geométricos y mecánicos de la tabla 3 empleados por D. Dominguez et al. en (24). La definición de cada uno de los parámetros se encuentra en (6o).

\section{DISEÑO DE LOS PÓRTICOS}

Los códigos de diseño estructural, describen diferentes procedimientos para el análisis de los edificios. Este trabajo considera 3 tipos de análisis:
Tabla 3. Valores numéricos de la geometría y comportamiento mecánico obtenidos en laboratorio. (24).

\begin{tabular}{|c|c|c|c|}
\hline Unidades & $\begin{array}{c}\text { Bloques } \\
\text { tradicionales }\end{array}$ & $\begin{array}{c}\text { Bloques } \\
\text { con aserrín }\end{array}$ & $\begin{array}{c}\text { Bloques } \\
\text { con viruta }\end{array}$ \\
\hline $\mathrm{t}(\mathrm{mm})$ & 191 & 191 & 191 \\
\hline $\mathrm{A}_{\mathrm{1}}\left(\mathrm{mm}^{2}\right)$ & 276970.21 & 276970.21 & 276970.21 \\
\hline $\mathrm{d}_{\mathrm{w}}(\mathrm{mm})$ & 5830.95 & 5830.95 & 5830.95 \\
\hline $\mathrm{b}_{\mathrm{w}}(\mathrm{mm})$ & 1457.74 & 1457.74 & 1457.74 \\
\hline $\mathrm{E}_{\mathrm{b}}\left(\mathrm{N} / \mathrm{mm}^{2}\right)$ & 36000 & 17964.07 & 16463.41 \\
\hline $\mathrm{b}(\mathrm{mm})$ & 192 & 192 & 192 \\
\hline $\mathrm{j}(\mathrm{mm})$ & 20 & 20 & 20 \\
\hline $\mathrm{E}_{\mathrm{m}}\left(\mathrm{N} / \mathrm{mm}^{2}\right)$ & 11709.30 & 4986.75 & 4429.08 \\
\hline $\mathrm{h}_{\mathrm{w}}(\mathrm{mm})$ & 3000 & 3000 & 3000 \\
\hline $\mathrm{E}_{\mathrm{j}}\left(\mathrm{N} / \mathrm{mm}^{2}\right)$ & 37150.60 & 37150.60 & 37150.60 \\
\hline $\mathrm{h}(\mathrm{mm})$ & 3400 & 3400 & 3400 \\
\hline $\mathrm{f}_{\mathrm{t}}(\mathrm{MPa})$ & 15 & 12 & 11.5 \\
\hline $\mathrm{f}_{\mathrm{m} \theta}(\mathrm{MPa})$ & 12.05 & 11.51 & 11.40 \\
\hline$\tau_{\mathrm{max}}(\mathrm{MPa})$ & 2.66 & 2.51 & 2.48 \\
\hline$\gamma_{\mathrm{p}}\left(\mathrm{kN} / \mathrm{m}^{3}\right)$ & 13.93 & 11.85 & 11.80 \\
\hline $\mathrm{z}(\mathrm{mm})$ & 852.81 & 1055.68 & 1087.43 \\
\hline$\lambda$ & 0.00180 & 0.00149 & 0.00144 \\
\hline$\theta\left({ }^{\circ}\right)$ & 30.96 & 30.96 & 30.96 \\
\hline $\mathrm{I}_{\mathrm{c}}\left(\mathrm{mm}{ }^{4}\right)$ & $675 \mathrm{e} 6$ & $675 \mathrm{e} 6$ & $675 \mathrm{e} 6$ \\
\hline & & & \\
\hline & & & \\
\hline & & & \\
\hline
\end{tabular}

a) Análisis estático utilizando las Normas de hormigón armado norteamericano ACI-318 y la Norma española EHE-o8, mediante las cuales, se han diseñado los elementos estructurales de los pórticos (vigas y pilares).

b) Análisis espectral utilizando las Normativas sismorresistentes europea (EC-8) y española (NCSE-02) para obtener las fuerzas sísmicas y el cortante basal de los pórticos. Los resultados obtenidos serán comparados con los resultados del análisis estatico no lineal (Push-over).

c) Análisis estático no lineal (Push-over), mediante el cual, se estima la capacidad última y plástica en términos de resistencia y desplazamiento para cada uno de los pórticos.

\subsection{Modelos de los muros de relleno}

En este apartado, se realizará un estudio comparativo de los cortantes basales máximos de cada uno de los pórticos analizados teniendo en cuenta la Normativa sismorresistente española (NCSE-02) y la Normativa europea (EC-8).

Los resultados obtenidos en la tabla 4, usando los espectros de las Normativas española y europea consideran un tipo de suelo de dureza medio (tipo II (NCSE-02) / Tipo C (EC-8)) y una aceleración sísmica moderada $\left(\mathrm{a}_{\mathrm{g}}=0.24 \mathrm{~g}\right)$, para un edificio de importancia normal $(S=1)$. Para todas las estructuras se han considerado un amortiguamiento del $5 \%$.

\subsection{Análisis estático no lineal (Push-over)}

El análisis estático no lineal de empuje incremental se emplea en la estimación de la capacidad horizontal máxima de una estructura, teniendo en cuenta la deformación y el contenido de frecuencia del movimiento de respuesta dinámica. Para la 
Tabla 4. Cortantes basales de los pórticos obtenidos de las Normativas

\begin{tabular}{|c|c|c|}
\hline Tipo de pórtico & NCSE-o2 (kN) & EC-8 (kN) \\
\hline $4-\mathrm{NW}$ & 762 & 832 \\
\hline $4-W T B$ & 1340 & 1456 \\
\hline $4-\mathrm{WAB}$ & 1226 & 1331 \\
\hline $4-\mathrm{WVB}$ & 1191 & 1294 \\
\hline $8-\mathrm{NW}$ & 992 & 1079 \\
\hline $8-\mathrm{WTB}$ & 1408 & 1532 \\
\hline $8-\mathrm{WAB}$ & 1284 & 1397 \\
\hline $8-\mathrm{WVB}$ & 1265 & 1377 \\
\hline $12-\mathrm{NW}$ & 1080 & 1172 \\
\hline $12-\mathrm{WTB}$ & 1472 & 1597 \\
\hline $12-\mathrm{WAB}$ & 1366 & 1483 \\
\hline $12-\mathrm{WVB}$ & 1339 & 1453 \\
\hline $16-\mathrm{NW}$ & 1113 & 1208 \\
\hline $16-W T B$ & 1457 & 1582 \\
\hline $16-W A B$ & 1401 & 1522 \\
\hline $16-W V B$ & 1391 & 1510 \\
\hline
\end{tabular}

evaluación y cálculos efectuados, se han utilizado las siguientes referencias (Antoniou y Pinho, 2004 (61); Ferracuti et al., $2009(62))$.

En la realización de los cálculos, la distribución de carga lateral no se mantiene constante, sino que se actualiza continuamente durante el análisis, de acuerdo con las formas modales y los factores de participación derivados del análisis de valor propio en cada paso del proceso de cálculo. Este método es multimodal, y explica el ablandamiento de la estructura, el alargamiento de su período y la modificación de las fuerzas de inercia debido a la amplificación espectral.

La actualización constante de los patrones de carga lateral de acuerdo con las propiedades modales que cambian constantemente el sistema, proporciona mejores estimaciones de respuesta que los métodos convencionales, especialmente en los casos en que existen irregularidades de resistencia o rigidez en la estructura y/o los efectos de modo más altos (por ejemplo; Crowley et al. 2008 (63)).

El algoritmo adaptativo que se implementa en SeismoStruct, es muy flexible y puede aceptar varios parámetros diferentes que se adaptan a los requisitos específicos de cada proyecto en particular. Son ejemplos, los métodos de combinación modal SRSS y CQC (Chopra, A. K., 1995 (64)).

Este análisis, permite calcular la capacidad de resistencia horizontal máxima de estructuras cuya respuesta dinámica no se ve afectada excesivamente por los niveles de deformación experimentados. Es decir, la distribución de fuerzas horizontales que simulan la respuesta dinámica se puede suponer constante. El análisis Push-over es uno de los cuatro procedimientos de análisis incorporados en FEMA 356/ASCE 41, que se utilizan en enfoques de diseño basados en el rendimiento. Para los lectores interesados, se puede encontrar una descripción detallada del método en $(25,65)$.

La metodología seguida en este trabajo, concentra las fallas de los pórticos en las rótulas plásticas que aparecen en las zonas cercanas a los nodos de cada elemento estructural (vigas y columnas). Los análisis se realizaron asumiendo una distribución de carga triangular. Este patrón de carga se incrementa proporcionalmente con un factor $(\lambda \cdot p)$ hasta que se alcanza la inestabilidad estructural. Para finalizar, en esta investigación se utiliza un control de respuesta correspondiente a un aumento en el desplazamiento de los nodos del piso superior.

Las figuras 4, 5, 6 y 7, muestran el comportamiento estático "no lineal" de los modelos analizados de 4, 8, 12 y 16 alturas respectivamente. Las gráficas representadas, permiten analizar el comportamiento elastoplástico de los pórticos previo al colapso. El comienzo de cada curva permite localizar el comportamiento elástico de las estructuras previo a una falla mayor. En la zona elástica, se apreciaron pequeñas diferencias entre los diferentes casos analizados. Sin embargo, a medida que aumenta la fuerza aplicada, las diferencias de desplazamiento son mayores entre los diferentes modelos para el mismo nivel de carga. Durante el aumento de la carga inicial, previa a la primera falla importante, son los muros de relleno los que soportan las fuerzas de corte de las estructuras. Estos elementos, aumentan la rigidez inicial de la estructura, haciendo fallar posteriormente los elementos estructurales principales (vigas y pilares). Esto se observa con una pérdida importante en la rigidez estructural inicial, una vez que se alcanza la resistencia máxima (tabla 5). Por otra parte, se puede ver como el comportamiento estructural de los pórticos con muros, una vez alcanzado el cortante máximo, tiende a igualarse con las estructuras sin muros. Esto se debe a que los muros colapsan antes que los elementos principales del pórtico (vigas y pilares), que son los que soportan la estructura.

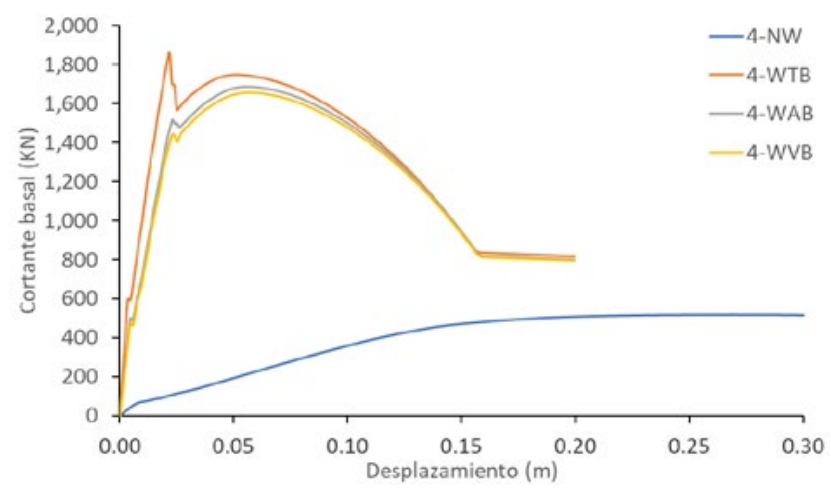

Figura 4. Curva de capacidad (cortante basal - deformación) para un pórtico de 4 plantas.

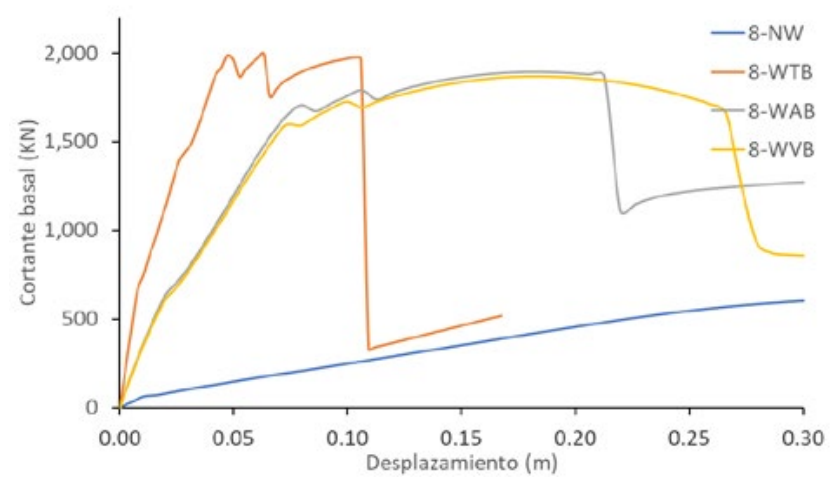

Figura 5. Curva de capacidad (cortante basal - deformación) para un pórtico de 8 plantas. 


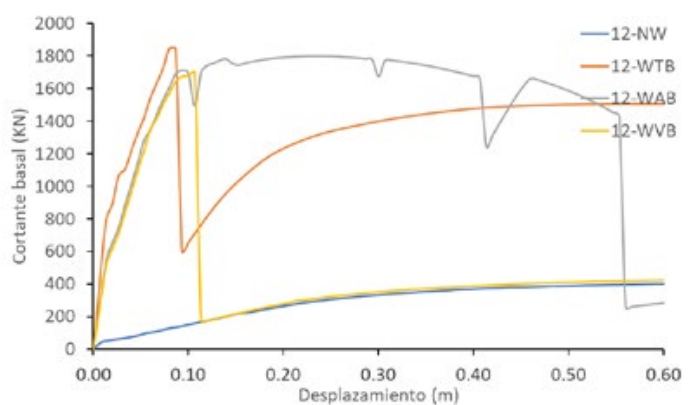

Figura 6. Curva de capacidad (cortante basal - deformación) para un pórtico de 12 plantas.

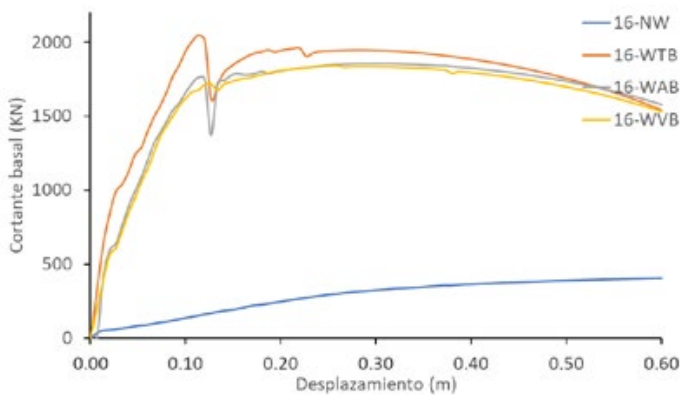

Figura 7. Curva de capacidad (cortante basal - deformación) para un pórtico de 16 plantas.

Tabla 5. Rigideces elásticas y efectiva de los pórticos.

\begin{tabular}{|c|c|c|c|}
\hline $\begin{array}{c}\text { Tipo de } \\
\text { pórtico }\end{array}$ & $\mathbf{K}_{\text {elas }}(\mathbf{k N} / \mathbf{m})$ & $\mathbf{K}_{\text {eff }}(\mathbf{k N} / \mathbf{m})$ & $\mathbf{K}_{\text {elas }} / \mathbf{K}_{\text {eff }}$ \\
\hline $4-\mathrm{NW}$ & 3147 & 1928 & 1.63 \\
\hline $4-\mathrm{WTB}$ & 88877 & 84898 & 1.04 \\
\hline $4-\mathrm{WAB}$ & 56256 & 30072 & 1.87 \\
\hline $4-\mathrm{WVB}$ & 53408 & 28550 & 1.87 \\
\hline $8-\mathrm{NW}$ & 1996 & 1275 & 1.56 \\
\hline $8-\mathrm{WTB}$ & 44042 & 31540 & 1.39 \\
\hline $8-\mathrm{WAB}$ & 19600 & 9820 & 1.99 \\
\hline $8-\mathrm{WVB}$ & 18776 & 9531 & 1.97 \\
\hline $12-\mathrm{NW}$ & 973 & 427 & 2.27 \\
\hline $12-\mathrm{WTB}$ & 25323 & 23070 & 1.10 \\
\hline $12-\mathrm{WAB}$ & 19728 & 7705 & 2.56 \\
\hline $12-\mathrm{WVB}$ & 19884 & 9582 & 2.07 \\
\hline $16-\mathrm{NW}$ & 803 & 354 & 2.26 \\
\hline $16-\mathrm{WTB}$ & 20826 & 18070 & 1.15 \\
\hline $16-\mathrm{WAB}$ & 15680 & 6190 & 2.53 \\
\hline $16-\mathrm{WVB}$ & 15537 & 7069 & 2.19 \\
\hline
\end{tabular}

La plasticidad en las curvas de capacidad se genera principalmente por las grietas producidas en el hormigón de los muros de relleno y a la plasticidad del refuerzo de acero existente en los elementos estructurales primarios. Estos efectos, se determinan por la formación de rótulas plásticas en cada uno de estos elementos. El mayor o menor número de rótulas que se forman determinará la longitud de estas curvas y la ductilidad de las edificaciones.

Las siguientes tablas (tabla 6 - 9), muestran los resultados estructurales obtenidos de las curvas de capacidad para cada uno de los pórticos. Los valores concernientes a la fuerza cortante de plastificación en la base del pórtico $\left(\mathrm{F}_{\mathrm{v}}\right)$ y al desplazamientos de plastificación en la parte superior del pórtico $\left(\boldsymbol{\delta}_{\mathrm{y}}\right)$, se han obtenido a través del sistema de áreas semejantes explicado en ATC40, mientras que los valores concernientes a la fuerza cortante última en la base del pórtico $\left(\mathrm{F}_{\mathrm{u}}\right)$ y al desplazamiento último en la parte superior del pórtico $\left(\boldsymbol{\delta}_{\mathrm{u}}\right)$ corresponden al punto de la curva donde la estructura sufre un descenso importante en su resistencia. Los valores de ductilidad $(\mu)$, se obtienen de la relación existente entre la deformación última $\left(\delta_{\mathrm{u}}\right)$ y la plástica $\left(\delta_{\mathrm{y}}\right)$.

Tabla 6. Deformaciones, cortante basal y ductilidad en un pórtico de 4 plantas.

\begin{tabular}{|c|c|c|c|c|c|}
\hline $\begin{array}{c}\text { Tipo de } \\
\text { bloque }\end{array}$ & $\boldsymbol{\delta}_{\mathbf{y}}(\mathbf{m})$ & $\boldsymbol{\delta}_{\mathbf{u}}(\mathbf{m})$ & $\begin{array}{c}\mathbf{F}_{\mathbf{y}} \\
(\mathbf{k N})\end{array}$ & $\begin{array}{c}\mathbf{F}_{\mathbf{u}} \\
(\mathbf{k N})\end{array}$ & $\boldsymbol{\mu}$ \\
\hline 4-NW & 0.149 & 0.268 & 469 & 517 & 1.80 \\
\hline 4-WTB & 0.018 & 0.022 & 1626 & 1858 & 1.20 \\
\hline 4-WAB & 0.026 & 0.056 & 1474 & 1685 & 2.14 \\
\hline 4-WVB & 0.027 & 0.058 & 1453 & 1657 & 2.13 \\
\hline
\end{tabular}

Tabla 7. Deformaciones, cortante basal y ductilidad en un pórtico de 8 plantas.

\begin{tabular}{|c|c|c|c|c|c|}
\hline $\begin{array}{c}\text { Tipo de } \\
\text { bloque }\end{array}$ & $\boldsymbol{\delta}_{\mathbf{y}}(\mathbf{m})$ & $\boldsymbol{\delta}_{\mathbf{u}}(\mathbf{m})$ & $\begin{array}{c}\mathbf{F}_{\mathbf{y}} \\
(\mathbf{k N})\end{array}$ & $\begin{array}{c}\mathbf{F}_{\mathbf{u}} \\
(\mathbf{k N})\end{array}$ & $\boldsymbol{\mu}$ \\
\hline 8-NW & 0.298 & 0.517 & 595 & 660 & 1.74 \\
\hline 8-WTB & 0.038 & 0.063 & 1669 & 1994 & 1.67 \\
\hline 8-WAB & 0.086 & 0.193 & 1674 & 1895 & 2.25 \\
\hline 8-WVB & 0.088 & 0.196 & 1643 & 1868 & 2.23 \\
\hline
\end{tabular}

Tabla 8. Deformaciones, cortante basal y ductilidad en un pórtico de 12 plantas.

\begin{tabular}{|c|c|c|c|c|c|}
\hline $\begin{array}{c}\text { Tipo de } \\
\text { bloque }\end{array}$ & $\boldsymbol{\delta}_{\mathbf{y}}(\mathbf{m})$ & $\boldsymbol{\delta}_{\mathbf{u}}(\mathbf{m})$ & $\begin{array}{c}\mathbf{F}_{\mathbf{y}} \\
(\mathbf{k N})\end{array}$ & $\begin{array}{c}\mathbf{F}_{\mathbf{u}} \\
(\mathbf{k N})\end{array}$ & $\boldsymbol{\mu}$ \\
\hline $12-\mathrm{NW}$ & 0.368 & 0.968 & 359 & 413 & 2.62 \\
\hline $12-\mathrm{WTB}$ & 0.056 & 0.080 & 1421 & 1846 & 1.43 \\
\hline $12-\mathrm{WAB}$ & 0.082 & 0.233 & 1608 & 1798 & 2.86 \\
\hline $12-\mathrm{WVB}$ & 0.071 & 0.177 & 1414 & 1696 & 2.49 \\
\hline
\end{tabular}

Tabla 9. Deformaciones, cortante basal y ductilidad en un pórtico de 16 plantas.

\begin{tabular}{|c|c|c|c|c|c|}
\hline $\begin{array}{c}\text { Tipo de } \\
\text { bloque }\end{array}$ & $\boldsymbol{\delta}_{\mathbf{y}}(\mathbf{m})$ & $\boldsymbol{\delta}_{\mathbf{u}}(\mathbf{m})$ & $\begin{array}{c}\mathbf{F}_{\mathbf{y}} \\
(\mathbf{k N})\end{array}$ & $\begin{array}{c}\mathbf{F}_{\mathbf{u}} \\
(\mathbf{k N})\end{array}$ & $\boldsymbol{\mu}$ \\
\hline $16-\mathrm{NW}$ & 0.479 & 1.260 & 384 & 446 & 2.63 \\
\hline $16-\mathrm{WTB}$ & 0.080 & 0.113 & 1664 & 2048 & 1.42 \\
\hline $16-\mathrm{WAB}$ & 0.106 & 0.300 & 1662 & 1857 & 2.83 \\
\hline $16-\mathrm{WVB}$ & 0.107 & 0.260 & 1661 & 1838 & 2.43 \\
\hline
\end{tabular}

\section{DISCUSIÓN DE LOS RESULTADOS}

El añadido de muros, aumenta significativamente la rigidez de los pórticos. El añadido de aditivos de madera en los bloques que conforman los muros disminuye la rigidez de los muros respecto de los bloques tradicionales, pero, sin embargo, aumenta la relación existente entre la rigidez plástica y elástica, prácticamente duplicándose.

Por otra parte, el añadido de aditivos de madera en la composición de los bloques rigidiza significativamente los 
pórticos, pero, sin embargo, produce un aumento en los desplazamientos elásticos y últimos respecto a los bloques tradicionales. Esto se traducirá en un mejor desempeño de la estructura frente a los movimientos sísmicos. Esta mejora, aumenta con el número de alturas de los pórticos.

La adición de aserrín o virutas de madera disminuye la densidad de los hormigones utilizados en la confección de los bloques. En este caso, la inclusión de aserrín y viruta disminuyó el peso de los bloques un $15 \%$, lo que se concluye en una reducción de las fuerzas sísmicas.

En este estudio, los valores obtenidos de los ensayos de laboratorio, se compararon con los requisitos establecidos en las Normas del código estructural chileno (NCh181). En este sentido, todos los materiales utilizados en este trabajo cumplen con los requisitos establecidos por el cemento NCh148 (1969), los agregados NCh163 (2013) y los estándares de características del agua NCh1498 (1982). La selección de este país es debido a la exigencia estructural de sus Reglamentos, haciendo cumplir los requisitos establecidos por otros reglamentos internacionales, como los Estados Unidos (ASTM 2004), México (NTC-M 2004), España (CTE DEB SE F 2006) o el Eurocódigo 6 (2005), entre otros.

En lo referente a los análisis estructurales efectuados a los pórticos, la resistencia de los mismos con el añadido de aditivos de madera disminuye la capacidad resistente de los mismos, pero mejora su comportamiento dúctil.

En este documento se ha estudiado el comportamiento estructural de los pórticos a través de análisis estáticos lineales (Normativa) y no lineales (Push-over). Desde el punto de vista del diseño sísmico, uno de los atributos más importantes de los materiales es su ductilidad, que refleja la capacidad de absorción y la disipación de energía que una estructura puede tomar antes de colapsar. En este sentido, un edificio debe disipar la energía que el movimiento del suelo le transfiere durante un terremoto. La forma más efectiva de hacerlo es mediante la deformación lateral y el deterioro local: daños internos donde la energía transferida a los materiales se convierte en calor. Si durante un episodio sísmico se alcanzara la deformación horizontal máxima estimada del análisis estático no lineal (Push-over), la estructura podría colapsar. Precisamente, la mayor o menor deformación que un terremoto exige en una estructura, se expresa en términos de ductilidad.

El comportamiento estático "no lineal" (Push-over) de los edificios con muros de relleno con bloques con aditivos para madera, mostró una mejora desde el punto de vista dúctil respecto de los muros ordinarios, haciendo estructuras más eficientes desde el punto de vista de disipar energía. En particular, una mayor flexibilidad en estos elementos disminuye la rigidez edificatoria, mejorando su rendimiento estructural. Además, cuanto más altos son los edificios, más significativa es la mejora.

La inclusión de muros en los pórticos, mejora significativamente la resistencia de los mismos desnudos. La diferencia de resistencias de los pórticos con muros de relleno respecto de los desnudos aumenta con la altura, produciéndose aumentos de 3 a 4 veces en los pórticos de 4 y 8 alturas y de 4 a 5 veces en los pórticos de 12 y 16 alturas.
La inclusión de muros de relleno de bloques tradicionales disminuye la ductilidad de los pórticos un 33\%, un 4\%, un $45 \%$ y un 46\% en los pórticos de 4, 8, 12 y 16 alturas respectivamente. Sin embargo, la inclusión de bloques con agregados de aserrín aumenta la ductilidad de las construcciones un $19 \%$, un 29\%, un 9\% y un 8\% en los pórticos de 4, 8, 12 y 16 alturas respectivamente.

Los cortantes basales últimos obtenidos de los cálculos normativos para los edificios sin muros son más restrictivos que los análisis Push-over realizados. Sin embargo, la inclusión de muros de relleno en los pórticos mejora el comportamiento sísmico de las construcciones, aumentando significativamente y haciendo mayores los cortantes basales respecto de los análisis Normativos. Además, ninguno de los pórticos con muros, teniendo en cuenta los cortantes obtenidos de las Normativas llega a plastificar.

Los desplazamientos máximos de los pórticos con muros disminuyen significativamente respecto de los pórticos desnudos. Sin embargo, la inclusión de muros de relleno con bloques con agregados de madera aumenta la capacidad de deformación en casi 3 veces las deformaciones respecto de los muros realizados con bloques tradicionales. De igual manera, las deformaciones plásticas aumentan con la inclusión de bloques con agregados de madera.

La utilización de bloques con agregados de madera en los muros de relleno aumenta la relación existente entre rigideces elásticas y efectivas. Este aumento es más significativo a medida que los pórticos aumentan de altura.

\section{CONCLUSIONES}

La resistencia de los pórticos se incrementa considerablemente con la utilización de muros de relleno.

La ductilidad de los pórticos disminuye con el uso de bloques tradicionales, sin embargo, el uso de los agregados de madera en la composición de los bloques, mejora significativamente esta propiedad. Esta ductilidad se incrementa con el aumento de alturas de las construcciones.

La normativa sismorresistente europea (EC-8) es levemente más restrictiva que la Normativa española (NCSE-02).

Los cortantes basales obtenidos en los análisis estáticos no lineales (Push-over) en los pórticos desnudos no cumple con los requisitos estructurales de las Normativas sismorresistentes. Sin embargo, la utilización de muros de relleno en los pórticos cumple con las exigencias de las Normativas sismorresistentes utilizadas en esta investigación.

La utilización de agregados de madera en los bloques de hormigón no afectaría significativamente en la resistencia de los pórticos con muros tradicionales. Sin embargo, las mejoras dúctiles de los bloques con agregados de madera, acompañadas de la reducción de peso de las edificaciones, podrían ser beneficiosas para el comportamiento sísmico de las edificaciones.

Finalmente, desde una perspectiva económica, la introducción de derivados de madera en el hormigón podría reducir el costo de las edificaciones, disminuyendo la cantidad de ma- 
terial y haciéndolas más eficientes estructuralmente desde el punto de vista sísmico.

El presente estudio es meramente exploratorio. A priori, los bloques con agregado de aserrín parecen exhibir leves mejoras en los comportamientos estructurales desde un punto de vista sísmico que los bloques con viruta. Sin embargo, el autor considera que estos resultados pueden variar cuando se tienen en cuenta otros porcentajes de aditivos, tipos de madera o granulometrías de madera. Además, también se deben analizar otras resistencias del hormigón y el acero utilizadas, así como otro número de pisos y/o configuraciones de edificios, para encontrar las dosis óptimas aplicables a una variedad más amplia de contextos. Como se puede observar, el número de combinaciones posibles para verificar es enorme. Por lo tanto, es previsible que sean necesarios varios estudios adicionales para cubrirlos a todos.

\section{REFERENCIAS / REFERENCES}

(1) Mukherjee, S. P., \& Vesmawala, G. (2013). Literature review on technical aspect of sustainable concrete. International Journal of Engineering Science Invention, 2(8), 1-9.

(2) Dominguez-Santos, D., Ballesteros-Perez, P., \& Mora-Melia, D. (2017). Structural resistance of reinforced concrete buildings in areas of moderate seismicity and assessment of strategies for structural improvement. Buildings, $7(4), 89$.

(3) De Landa, M. (1997). A thousand years of nonlinear history.

(4) Mehta, P. K., \& Monteiro, P. J. (2017). Concrete microstructure, properties and materials.

(5) Thorat, P. K., Papal, M., Kacha, V., Sarnobat, T., \& Gaikwad, S. (2015). Hollow concrete blocks-A new trend. International Journal of Engineering \& Research, 5(5), 9-26.

(6) Ahmad, R., Malik, M. I., Jan, M. U., Ahmad, P., Seth, H., \& Ahmad, J. (2014). Brick Masonry and Hollow Concrete Block Masonry-A Comparative Study. International Journal of Civil and Structural Engineering Research (IJCSER), 1(1), 14-21.

(7) Maroliya, M. K. (2001). Load carrying capacity of hollow concrete block masonry column. IOSR J. Eng.(IOSRJEN), 2(10), 5-8.

(8) Avila, L., Vasconcelos, G., Lourenço, P. B., Mendes, N., Alves, P., \& Costa, A. C. (2012). Seismic response analysis of concrete block masonry buildings: An experimental study using shaking table.

(9) Frasson Jr, A., Casali, J. M., Oliveira, A. L., \& Prudêncio Jr, L. R. (2012, June). A Mix design methodology for concrete block units. In Proceedings of the 15th International Brick and Block Masonry Conference, Florianapolis, Brazil (pp. 3-6).

(10) Rathi, S. O., \& Khandve, P. V. (2015). AAC block-A new eco-friendly material for construction. International Journal of Advance Engineering and Research Development, 2(4), 410-414.

(11) Mindess, S., \& Vondran, G. (1988). Properties of concrete reinforced with fibrillated polypropylene fibres under impact loading. Cement and Concrete Research, 18(1), 109-115.

(12) Bayasi, Z., \& McIntyre, M. (2002). Application of fibrillated polypropylene fibers for restraint of plastic shrinkage cracking in silica fume concrete. Materials Journal, 99(4), 337-344.

(13) Ashour, S. A., \& Wafa, F. F. (1993). Flexural behavior of high-strength fiber reinforced concrete beams. Structural Journal, $90(3), 279-287$.

(14) Oh, B. H. (1992). Flexural analysis of reinforced concrete beams containing steel fibers. Journal of structural engineering, 118(10), 2821-2835.

(15) Sabir, B. B., Wild, S., \& Bai, J. (2001). Metakaolin and calcined clays as pozzolans for concrete: a review. Cement and concrete composites, 23(6), 441-454.

(16) Quaranta, N., Caligaris, M., López, H., Unsen, M., \& Di Rienzo, H. (2008). Adición de aserrines de descarte en la producción de mampuestos cerámicos. In Actas del Octavo Congreso Internacional de Metalurgia y Materiales.

(17) Nili, M., Sasanipour, H., \& Aslani, F. (2019). The effect of fine and coarse recycled aggregates on fresh and mechanical properties of self-compacting concrete. Materials, 12(7), 1120.

(18) Xie, J., Zhao, J., Wang, J., Wang, C., Huang, P., \& Fang, C. (2019). Sulfate resistance of recycled aggregate concrete with GGBS and fly ash-based geopolymer. Materials, 12(8), 1247.

(19) Liu, W., Cao, W., Zhang, J., Qiao, Q., \& Ma, H. (2016). Seismic performance of composite shear walls constructed using recycled aggregate concrete and different expandable polystyrene configurations. Materials, 9(3), 148.

(20) Zhu, L., Dai, J., Bai, G., \& Zhang, F. (2015). Study on thermal properties of recycled aggregate concrete and recycled concrete blocks. Construction and Building Materials, 94, 620-628.

(21) Miličević, I., Bjegović, D., \& Siddique, R. (2015). Experimental research of concrete floor blocks with crushed bricks and tiles aggregate. Construction and Building materials, 94, 775-783.

(22) Pastor, J. M., García, L. D., Quintana, S., \& Peña, J. (2014). Glass reinforced concrete panels containing recycled tyres: Evaluation of the acoustic properties of for their use as sound barriers. Construction and Building Materials, 54, 541-549.

(23) Ergün, A. (2011). Effects of the usage of diatomite and waste marble powder as partial replacement of cement on the mechanical properties of concrete. Construction and building materials, 25(2), 806-812.

(24) Dominguez-Santos, D., Mora-Melia, D., Pincheira-Orellana, G., Ballesteros-Pérez, P., \& Retamal-Bravo, C. (2019). Mechanical properties and seismic performance of wood-concrete composite blocks for building construction. Materials, 12(9), 1500.

(25) López-Almansa, F., Domínguez, D., \& Benavent-Climent, A. (2013). Vulnerability analysis of RC buildings with wide beams located in moderate seismicity regions. Engineering structures, 46, 687-702.

(26) Domínguez, D., López-Almansa, F., \& Benavent Climent, A. (2014). Comportamiento, para el terremoto de Lorca de 1105-2011, de edificios de vigas planas proyectados sin tener en cuenta la acción sísmica.

(27) Page, J., Djelal, C., \& Vanhove, Y. (2020). Optimisation of vibrocompaction process for wood-based concrete blocks. The International Journal of Advanced Manufacturing Technology, 109(3), 1189-1204. 
(28) Bootle, K. R. (1983). Wood in Australia. Types, properties and uses. McGraw-Hill book company..

(29) Filiatrault, A., \& Folz, B. (2002). Performance-based seismic design of wood framed buildings. Journal of Structural Engineering, 128(1), 39-47.

(30) Alengaram, U. J., Salam, A., Jumaat, M. Z., Jaafar, F. F., \& Saad, H. B. (2011). Properties of high-workability concrete with recycled concrete aggregate. Materials Research, 14(2), 248-255.

(31) ASTM D143 - 09. (2009) Standard Test Methods for Small Clear Specimens of Timber. USA Standard.

(32) Wangaard, F. F., Kellogg, R. M., \& Brinkley, A. W. (1966). Variation in wood and fiber characteristics and pulp-sheet properties of slash pine. Tappi, 49(6), 263.

(33) RFS Hearmon - Forest Products Research, (1948). The elasticity of wood and plywood.

(34) Carle, J. y Holmgren, P. (2008). Wood from planted forests: A global outlook 2005-2030. For. Prod. J. 58, 6.

(35) Raga, F. (2009). The Chilean forestry sector and associated risks. Trébol, 51, 10-19.

(36) González, P. (2006). Disponibilidad de residuos madereros provenientes de la industria primaria de la madera para uso energético XI Región de Aysén. In Proceedings of the seminar Hacia una Politica Nacional de Bioenergía, Aysen, Chile.

(37) NCh 1019 Of 09. (2009). Hormigón. Determinación de la Docilidad. Método del Asentamiento del cono de Abrams. Instituto Nacional de Normalización: Santiago, Chile. (Chilean Standard).

(38) NCh 1037 Of77, 1977 (1997). Hormigón. Ensayo de Compresión de Probetas Cúbicas y Cilíndricas. Instituto Nacional de Normalización: Santiago, Chile. (Chilean Standard).

(39) NCh 163, 2013. (2013). Áridos Para Morteros y Hormigones. Requisitos. Instituto Nacional de Normalización: Santiago, Chile. (Chilean Standard).

(40) NCh 165 Of 77. (2009). Áridos Para Morteros y Hormigones-Tamizado y Determinación de la Granulometría. Instituto Nacional de Normalización: Santiago, Chile. (Chilean Standard).

(41) Real Decreto 1247. (2008). Instrucción de Hormigón Estructural. EHE o8; Spain. (Spanish Standard).

(42) CTN 140. (2010). Eurocodigos Estructurales. Una Norma Española (UNE). (Spanish Standard).

(43) Crisafulli, F. J., Carr, A. J., \& Park, R. (2000). Analytical modelling of infilled frame structures. Bulletin of the New Zealand Society for Earthquake Engineering, 33(1), 30-47.

(44) Lizárraga, J. F., \& Pérez Gavilán, J. J. (2015). Modelación no lineal de muros de mampostería empleando elementos de contacto. Ingeniería sísmica, (93), 41-59.

(45) Smyrou, E., Blandon, C., Antoniou, S., Pinho, R., \& Crisafulli, F. (2011). Implementation and verification of a masonry panel model for nonlinear dynamic analysis of infilled RC frames. Bulletin of Earthquake Engineering, 9(5), 1519-1534.

(46) Wararuksajja, W., Srechai, J., \& Leelataviwat, S. (2020). Seismic design of RC moment-resisting frames with concrete block infill walls considering local infill-frame interactions. Bulletin of Earthquake Engineering. 18(14), 6445-6474.

(47) Di Domenico, M., Ricci, P., \& Verderame, G. M. (2019). Predicting the out-of-plane seismic strength of unreinforced masonry infill walls. Journal of Earthquake Engineering, 1-38.

(48) SeismoSoft. (2020). A Computer Program for Static and Dynamic Nonlinear Analysis of Framed Structures. Available online: http://www.seismosoft.com.

(49) CTE DEB SE F, (2006). Documento Básico. Código Técnico de la Edificación. Seguridad Estructural: Fabrica. Ministerio de Fomento: Madrid, Spain. (Spanish Standard).

(50) Mander, J. B., Priestley, M. J., \& Park, R. (1988). Theoretical stress-strain model for confined concrete. Journal of structural engineering, 114(8), 1804-1826.

(51) Bosco, M., Ferrara, E., Ghersi, A., Marino, E. M., \& Rossi, P. P. (2016). Improvement of the model proposed by Menegotto and Pinto for steel. Engineering Structures, 124, 442-456.

(52) Spacone, E., \& Filippou, F. (1996, June). Flexibility-based frame models for nonlinear dynamic analysis. In Proceedings of the 11th World Conference on Earthquake Engineering, Acapulco, Mexico (pp. 23-28).

(53) Scott, M. H., \& Fenves, G. L. (2006). Plastic hinge integration methods for force-based beam-column elements. Journal of Structural Engineering, 132(2), 244-252.

(54) Scott, M. H., Fenves, G. L., McKenna, F., \& Filippou, F. C. (2008). Software patterns for nonlinear beam-column models. Journal of Structural Engineering, 134(4), 562-571.

(55) Gavin, H. (2001). Numerical integration for structural dynamics. Department of Civil and Environmental Engineering, Duke University: Durham, NC, USA.

(56) Haldar, A., \& Mahadevan, S. (2000). Probability, reliability, and statistical methods in engineering design. John Wiley.

(57) Mergos, P. E., \& Kappos, A. J. (2015). Estimating fixed-end rotations of reinforced concrete members at yielding and ultimate. Structural Concrete, 16(4), 537-545.

(58) Priestley, M. N., Calvi, G. M., \& Kowalsky, M. J. (2007). Displacement-based seismic design of structures. IUSS press.

(59) Crisafulli, F. J., \& Carr, A. J. (2007). Proposed macro-model for the analysis of infilled frame structures. Bulletin of the New Zealand Society for Earthquake Engineering, 4O(2), 69-77.

(6o) Crisafulli, F. J. (1997). Seismic behaviour of reinforced concrete structures with masonry infills.

(61) Antoniou, S., \& Pinho, R. (2004). Development and verification of a displacement-based adaptive pushover procedure. Journal of earthquake engineering, 8(05), 643-661.

(62) Ferracuti, B., Pinho, R., Savoia, M., \& Francia, R. (2009). Verification of displacement-based adaptive pushover through multi-ground motion incremental dynamic analyses. Engineering Structures, 31(8), 1789-1799.

(63) Crowley, H., Borzi, B., Pinho, R., Colombi, M., \& Onida, M. (2008). Comparison of Two Mechanics-Based Methods for Simplified Structural Analysis in Vulnerability Assessment. Advances in civil Engineering.

(64) Chopra, A. K. (1995). Dynamics of structures theory and.

(65) Paulay, T., \& Priestley, M. N. (1992). Seismic design of reinforced concrete and masonry buildings. 